\title{
Multiple Supernova Explosions in a Forming Galaxy
}

\author{
Masao Mori ${ }^{1,4}$, Masayuki Umemura ${ }^{2}$, and Andrea Ferrara ${ }^{3}$ \\ ${ }^{1}$ Institute of Natural Sciences, Senshu University, Kawasaki, Kanagawa, 214-8580, Japan \\ 2 Center for Computational Physics, University of Tsukuba, Tsukuba, Ibaraki, 305-8577, Japan \\ ${ }^{3}$ SISSA/International School for Advanced Studies, 34014 Trieste, Italy \\ ${ }^{4}$ E-mail: mmori@isc.senshu-u.ac.jp
}

Received 2003 October 1, accepted 2004 March 11

\begin{abstract}
Ultra-high resolution hydrodynamic simulations using $1024^{3}$ grid points are performed of a very large supernova burst in a forming galaxy, with properties similar to those inferred for Lyman Break Galaxies (LBGs). Explosions produce kpc-sized expanding hot bubbles enclosed by cool, dense shells, and the engulfed gas is polluted with freshly-synthesised heavy elements. We show that the resultant inhomogeneous mixing produces a large spread $([\mathrm{Fe} / \mathrm{H}] \approx-1$ to -5$)$ of metallicities, which affects the subsequent galactic chemical evolution and leaves its imprint on metal-poor stars. By combining a spectral synthesis model with the numerical results, we predict Ly $\alpha$ emission from such galaxy at $z=3$. We find that the simulated galaxy, whose peak star formation rate is $\approx 200 \mathrm{M}_{\odot} \mathrm{yr}^{-1}$, produces a Ly $\alpha$ luminosity $L_{\alpha}=9.7 \times 10^{42} \mathrm{erg} \mathrm{s}^{-1}$. This value favorably matches the observed one, but some discrepancies are left for the Ly $\alpha$ line width, the metallicity, and X-ray properties. Since the results of the simulation is applicable only at the very early epoch of the galaxy formation, the metallicity is still lower than that of LBGs. However, the analysis presented here demonstrates a way to enable a systematic comparison with observational data.

It is concluded that LBGs are optimal objects to scrutinise the early self-enrichment in forming galaxies. In the future, the predicted bubbly structure carved by $\mathrm{SNe}$ may be directly detected by high resolution observations with JWST.
\end{abstract}

Keywords: cosmology: theory — galaxies: formation — galaxies: high-redshift — galaxies: starburst — hydrodynamics — stars: Population II — supernovae: general

\section{Introduction}

Our understanding of galaxy formation has greatly deepened in last decade due to multi-wavelength observations of star-forming galaxies at high redshifts. Optical observations have revealed the presence of a number of Lyman break galaxies (LBGs) at redshifts of $3 \lesssim z \lesssim 5$ (Steidel et al. 1996; Steidel et al. 1999; Pettini et al. 2001; Giavalisco 2002) as well as Ly $\alpha$ emitters at $3 \lesssim z \lesssim 6$ (Dey et al. 1998; Weyman et al. 1998; Rhoads et al. 2000; Hu et al. 2002; Ajiki et al. 2002; Dawson et al. 2002; Fujita et al. 2003; Kodaira et al. 2003). A good fraction of LBGs exhibit Ly $\alpha$ emission lines strong enough to be detected by narrow band observations (Shapley et al. 2003); also bright Ly $\alpha$ 'blobs' which are associated but not centred on known LBG have been detected, with a Ly $\alpha$ luminosity of $\sim 10^{43} \mathrm{erg} \mathrm{s}^{-1}$ at $z=3.09$ (Steidel et al. 2000). LBGs are usually characterised by compact cores (some with multiple components) of $5-8 \mathrm{kpc}$ and often surrounded by more diffuse, asymmetric halos (Steidel et al. 1996; Giavalisco et al. 1996), whereas Ly $\alpha$ blobs extended for about $\sim 100 \mathrm{kpc}$. LBGs are likely to be young galaxies experiencing major episodes of star formation: stellar age determinations yield $30 \mathrm{Myr} \lesssim t \lesssim 1 \mathrm{Gyr}$ (Papovich, Dickinson, \& Ferguson 2001), or $0.1 \mathrm{Gyr} \lesssim t \lesssim 1 \mathrm{Gyr}$
(Pettini et al. 2001) and the inferred star formation rate (SFR) is $\approx 100 \mathrm{M}_{\odot} \mathrm{yr}^{-1}$ (Teplitz et al. 2000; Shapley et al. 2001; Nandra et al. 2002; Ferguson, Dickinson, \& Papovich 2002). Since LBGs are quite young, they could hold direct interpretable information on the early chemical enrichment of galaxies, contrary to present-day galaxies which have undergone intense interstellar medium (ISM) recycling, thus erasing most of the early chemical history.

So far, the theoretical models of galactic chemical evolution have often assumed the homogeneous ISM (one-zone model), with the instantaneous and perfect mixing of heavy elements synthesised in supernovae ( $\mathrm{SNe}$ ). However, the energy input and metal ejection by $\mathrm{SNe}$ are likely to proceed in an inhomogeneous fashion (Tsujimoto \& Shigeyama 1998; Argast et al. 2000). Thus, simulations that can resolve $\mathrm{SN}$ remnants are required to properly model the chemical evolution of primordial galaxies. In this paper, we perform ultra-high resolution hydrodynamic simulations of a very large burst of multiple SN explosions in a forming galaxy. Also, by incorporating spectro-photometric modelling with the simulations, the results can be directly compared to the observations on high-redshift forming galaxies in a similar way to Mori et al. (1997) and Mori, Yoshii, \& Nomoto 
(1999). Here, we investigate the chemical enrichment pattern and the Ly $\alpha$ emission properties of such objects to check the hypothesis that they could well represent LBGs and Ly $\alpha$ blobs at high redshifts. Finally, the X-ray emission property predicted by the simulation and some implications for metal-poor halo stars are discussed.

\section{Model}

We define a fiducial model for the bright-end of LBGs at redshift $z=3$, in which the gas mass is initially assumed to be $M_{\mathrm{g}}=9.5 \times 10^{11} \mathrm{M}_{\odot}$, and the total mass including dark matter halo is $M_{\mathrm{t}}=3.7 \times 10^{12} \mathrm{M}_{\odot}$. The baryonic to dark matter halo has been increased by a factor of about two with respect to the cosmological value, ${ }^{1}$ to account for possible bias effects in rapidly cooling regions. According to the general concept of hierarchical scenarios of galaxy formation we build up the galaxy as an assemblage of a large number of subgalactic structures. Such systems are assumed to be already virialised and have a NFW density profile, $\rho_{\mathrm{NFW}}(r) \propto r^{-1}(r+a)^{-2}$ (Navarro, Frenk, \& White 1997), while by considering the surface density profiles of LBGs the galaxy as a whole is set to be a pre-virialised system with a shallower profile with the spherically averaged density of $\rho_{\mathrm{d}}(r) \propto \rho_{\mathrm{NFW}}(r)^{1 / 2}$ in a virial radius of $106 \mathrm{kpc}$. The small condensations have a halo mass $1.4 \times 10^{9} \mathrm{M}_{\odot}$ within a virial radius $r_{\mathrm{s}}=8.60 \mathrm{kpc}$. (The mass spread of these small systems is not taken into account, which is left in the future study.)

We focus on the first $50 \mathrm{Myr}$ of the star formation activity in light of the inferred age of LBGs. The star formation rate $\operatorname{SFR}(t)$ is assumed to be linearly proportional to the total gas mass; when integrated over the whole system it is $\operatorname{SFR}(t)=204 \exp \left(-t / t_{\star}\right) \mathrm{M}_{\odot} \mathrm{yr}^{-1}$ with the time scale $t_{\star}=4.6 \mathrm{Gyr}$. Because of the short timescale of our simulation, the SFR is essentially constant $\sim 200 \mathrm{M}_{\odot} \mathrm{yr}^{-1}$ which is consistent with that derived from observations (Shapley et al. 2001). The mass of individual stars and the formation epoch are determined by randomly sampling the initial mass function (IMF). It is not likely that LBGs are dominated by first-generation stars (Papovich et al. 2001). Thus, in the present study, we adopt Salpeter's IMF with upper and lower mass cutoffs of $120 \mathrm{M}_{\odot}$ and $0.1 \mathrm{M}_{\odot}$, respectively. A star more massive than $8 \mathrm{M}_{\odot}$ is assumed, after the main sequence lifetime, to undergo a Type II SN explosion, releasing a total energy of $10^{51} \mathrm{erg}$ and expel synthesised heavy elements tabulated by Tsujimoto et al. (1995). In total $7.53 \times 10^{7} \mathrm{SN}$ explosions occur in the whole galaxy.

The gas dynamics is pursued by a three-dimensional hydrodynamic scheme with $1024^{3}$ Cartesian grids. The simulation box has a (physical) size of $212 \mathrm{kpc}$ and the spatial resolution is $195 \mathrm{pc}$. We use a tracer field advected with the same algorithm as the density in order to follow

\footnotetext{
${ }^{1}$ Throughout this paper we assume a $\Lambda$ CDM cosmology with $\Omega_{\mathrm{M}}=0.3$, $\Omega_{\Lambda}=0.7$, a Hubble constant of $h=H_{0} /\left(100 \mathrm{~km} \mathrm{~s}^{-1} \mathrm{Mpc}^{-1}\right)=0.7$ and $\Omega_{b} h^{2}=0.02$.
}

the metallicity of the gas. Since the time variation of dark matter halo potential is small in the first $50 \mathrm{Myr}$, we assume the gravitational potential to be constant. Also, the gas is assumed to be optically thin and in collision-ionization equilibrium. Radiative cooling losses are calculated selfconsistently using the metallicity-dependent curves of Sutherland \& Dopita (1993). The set of basic equations are numerically solved by a parallel version of the Astrophysical Fluid Dynamics (AFD2) scheme (see Mori, Ferrara, \& Madau 2002; M. Mori, in preperation).

\section{Results}

Figure 1 shows the numerical results at $50 \mathrm{Myr}$ by the snapshots in a slice along the $X-Y$ sectional plane. Figures $1 \mathrm{a}$ and $1 \mathrm{~b}$ show the density and temperature distributions, respectively. After the first massive stars explode, the gas temperature increases up to about $10^{8} \mathrm{~K}$ locally, and expanding hot bubbles of $\mathrm{kpc}$ dimensions are produced; they are enclosed by cooled, dense shells. Subsequent SN explosions further accelerate the expansion of hot bubbles and the ambient gas is continuously swept up by the shells; the gas density increases further owing to the enhanced cooling in the dense shells. Since in the outer regions the density of the ISM is low, the bubble expansion is faster and $\mathrm{SN}$-driven shock waves quickly collide with each other to generate super-bubbles of $\sim 10 \mathrm{kpc}$, which are surrounded by high-density $\left(\gtrsim 0.1 \mathrm{~cm}^{-3}\right)$, cool $\left(\sim 10^{4} \mathrm{~K}\right)$ shells.

Figure 1c shows the distribution of metallicity, here represented by iron abundance. The gas in the vicinity of $\mathrm{SNe}$ is polluted with synthesised heavy elements ejected from $\mathrm{SNe}$, but a large amount of the gas still retains low metallicity. The interactions of expanding hot bubbles give rise to a complex structure in the inner regions, where a metal-rich gas $[\mathrm{Fe} / \mathrm{H}] \gtrsim-2.3$ coexists with an almost primordial gas. They are separated from each other by cool shells. Interestingly, the outer regions tend to have higher metallicity compared with inner regions. This is because the metal-enriched super-bubbles are less diluted with metal-deficient gas in the outer low-density regions.

The distribution of the line-of-sight velocity is shown in Figure 1d. Since a part of gas has a velocity higher than $500 \mathrm{~km} \mathrm{~s}^{-1}$, which exceeds the escape velocity of this galaxy $\left(472 \mathrm{~km} \mathrm{~s}^{-1}\right)$, it might escape from the galaxy's potential well. However, the deep gravitational potential of a massive galaxy like the one we study here inhibits a significant mass loss from the system. Winds are instead much more effective in less massive galaxies (typically $M_{t} \lesssim 10^{10} \mathrm{M}_{\odot}$, Ferrara, Pettini, \& Shchekinov 2000), thus representing an efficient mechanism to distribute the heavy elements over cosmological volumes (Mori, Ferrara, \& Madau 2002).

Furthermore, we compute the emission properties of the galaxy assuming for an optically thin gas in collisional ionization equilibrium and using the MAPPINGS III code by Sutherland \& Dopita (1993). In practice, to obtain the SED of the system we sum up the SED of each 

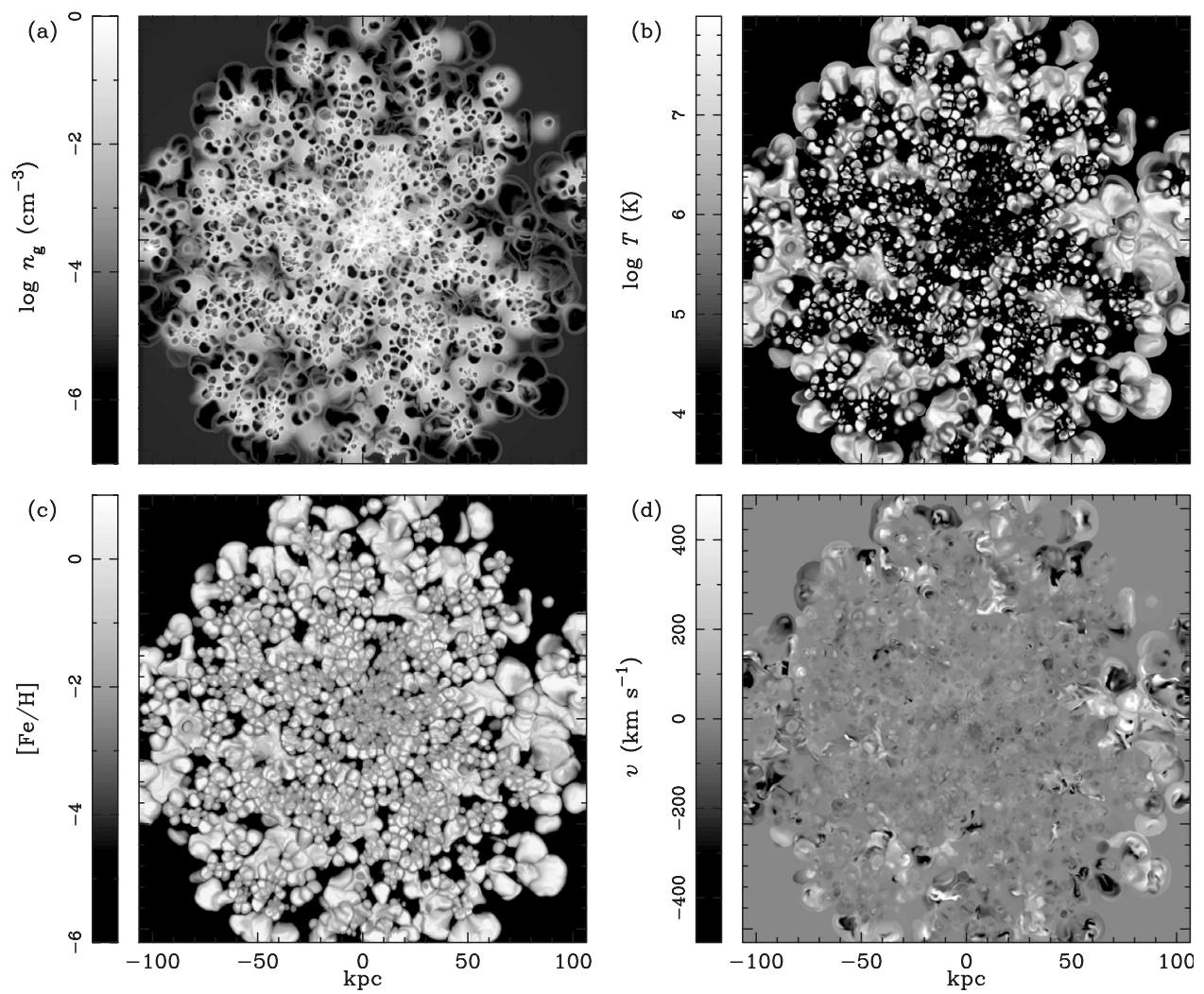

Figure 1 Distributions of physical quantities in a slice along the $X-Y$ sectional plane at the elapsed time of $50 \mathrm{Myr}$. (a) Snapshot of the logarithmic density, (b) the logarithmic temperature, (c) the iron abundance $[\mathrm{Fe} / \mathrm{H}]$, and (d) the line-of-sight velocity component.

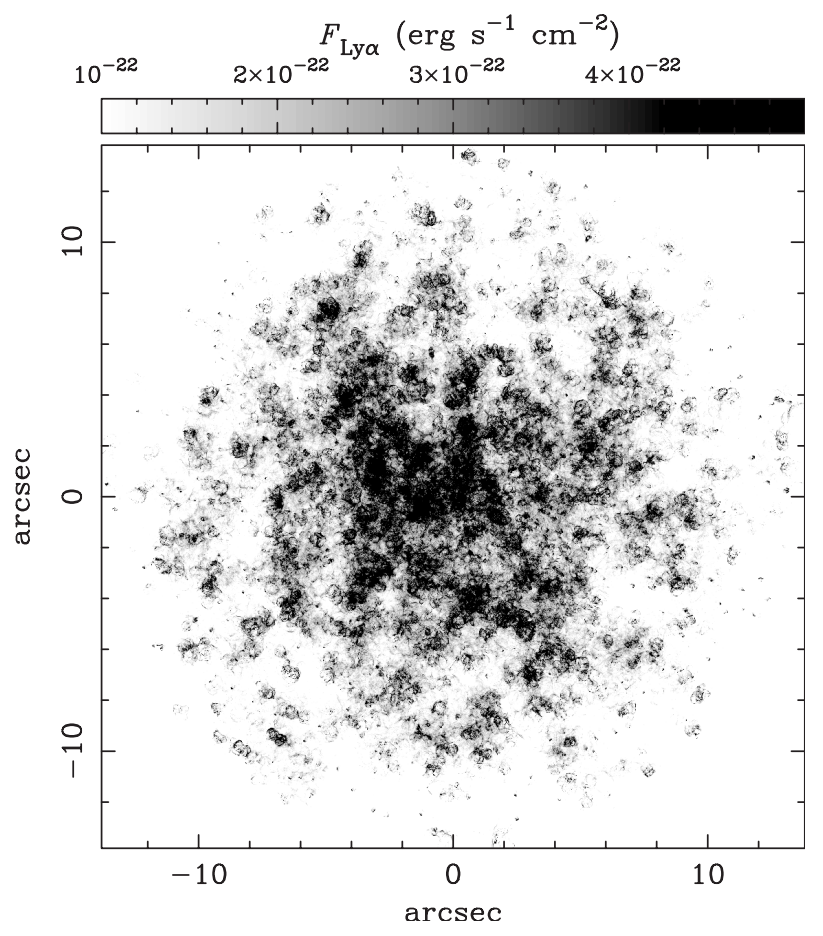

Figure 2 The projected distribution of the resultant $\operatorname{Ly} \alpha$ emission, where the angular resolution is $2.7 \times 10^{-2}$ arcsec (corresponding to the numerical resolution, $195 \mathrm{pc}$ ).

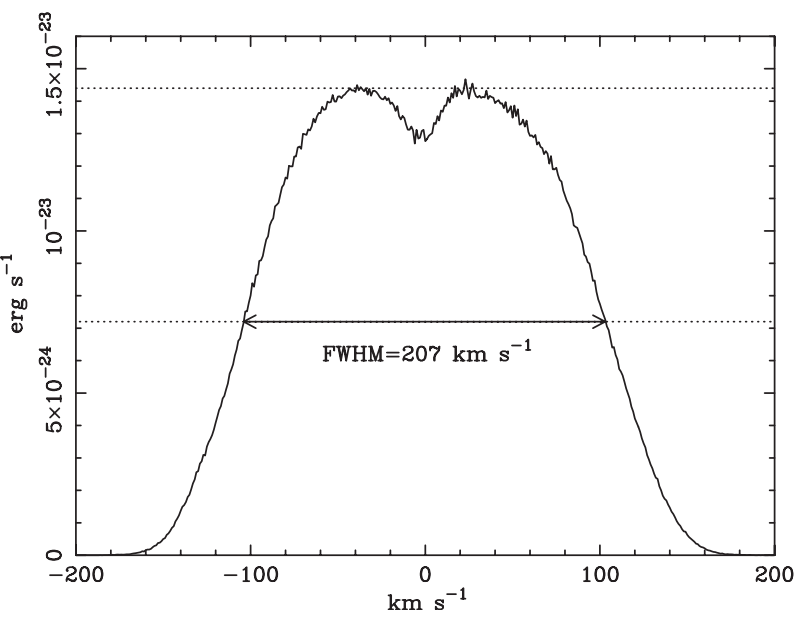

Figure 3 The line profile of $\operatorname{Ly} \alpha$ emission from the simulated galaxy. The full width at half maximum is $207 \mathrm{~km} \mathrm{~s}^{-1}$.

grid point. Figure 2 shows the projected distribution of the resultant Ly $\alpha$ emission, where the angular resolution is $2.7 \times 10^{-2}$ arcsec (corresponding to the numerical resolution, $195 \mathrm{pc}$ ). The total flux of $\mathrm{Ly} \alpha$ emission is $1.2 \times 10^{-16} \mathrm{erg} \mathrm{s}^{-1} \mathrm{~cm}^{-2}$ and the corresponding luminosity is $9.7 \times 10^{42} \mathrm{erg} \mathrm{s}^{-1}$, which comes mainly from 
high-density cooling shells. This Ly $\alpha$ luminosity is just comparable to $\sim 10^{43} \mathrm{erg} \mathrm{s}^{-1}$ of extended Ly $\alpha$ blobs and Ly $\alpha$ emitters at higher-redshift $z>5$. Figure 3 shows the line profile of $\operatorname{Ly} \alpha$ emission from the simulated galaxy. A twin peak emerges due to the superposition of the expanding SN shells. The full width at half maximum (FWHM) of Ly $\alpha$ emission is $207 \mathrm{~km} \mathrm{~s}^{-1}$, which falls short of the FWHM of Ly $\alpha$ emission for observed LBGs, $450 \pm 150 \mathrm{~km} \mathrm{~s}^{-1}$, or that for Ly $\alpha$ emitters, $\approx 350 \mathrm{~km} \mathrm{~s}^{-1}$ (Ajiki et al. 2002; Kodaira et al. 2003). Ly $\alpha$ extinction due to dust is negligible: for the average gas column density, $N_{\mathrm{H}}=5.6 \times 10^{21} \mathrm{~cm}^{-2}$, and metallicity in the simulation, we have $E(B-V)=$ $\left(N_{\mathrm{H}} / 9.2 \times 10^{21} \mathrm{~cm}^{-2}\right) \times 10^{[\mathrm{Fe} / \mathrm{H}]}=3.06 \times 10^{-3}$. Then, using equation (20) of Pei (1992) and $A_{\mathrm{B}}=1.2 \times 10^{-2}$, we find $\tau_{\mathrm{Ly} \alpha}=3.07 \times 10^{-2}$. In the future, the detailed bubbly structure seen in Figure 2 may be directly detected by high resolution observations $(\sim 0.1 \mathrm{arcsec})$, of redshifted $\mathrm{H}_{\alpha}$ emission $\left(1.5 \times 10^{41} \mathrm{erg} \mathrm{s}^{-1}\right)$, with the James Webb Space Telescope.

\section{Discussion}

We have studied the inhomogeneous mixing of heavy elements in the early phase of galaxy evolution by ultrahigh resolution hydrodynamic simulations. The X-ray emission properties are also calculated to compare the observed ones. For example, Chandra deep X-ray survey (Brandt et al. 2001; Nandra et al. 2002) of the Hubble Deep Field-North region have derived a mean X-ray luminosity $\approx(0.7-3.4) \times 10^{41} \mathrm{erg} \mathrm{s}^{-1}$ for the sample of LBGs found. Our simulation predicts that the total flux of $\mathrm{X}$-ray emission in the $0.2-2 \mathrm{keV}$ band $(0.8-8 \mathrm{keV}$ at $z=3$ ) is $1.5 \times 10^{-19} \mathrm{erg} \mathrm{s}^{-1} \mathrm{~cm}^{-2}$ and the corresponding luminosity is $1.1 \times 10^{40} \mathrm{erg} \mathrm{s}^{-1}$. This value is an order of magnitude lower than the observed luminosity. It may imply that the observed X-ray arises not only from diffuse hot gases but also from a combination of other sources like high-mass X-ray binaries and low-luminosity active galactic nuclei, which are not included in the present simulation. On the other hand, we must pay attention, however, the result of our simulation presented here is applicable only at the very early epoch of the galaxy formation. A continuous energy input by $\mathrm{SNe}$ after the initial starburst can increase the amount of hot gas and enhance, therefore, the X-ray luminosity. In a series of forthcoming studies, we will investigate the subsequent evolution of the proto-galaxies and their X-ray luminosity.

The volume-averaged metallicity of the gas is found to be $[\mathrm{Fe} / \mathrm{H}]_{\mathrm{V}}=-2.30$, while the density-weighted metallicity is $[\mathrm{Fe} / \mathrm{H}]_{\rho}=-2.51$. On the other hand, if one derives the mean metallicity based on X-ray flux, $[\mathrm{Fe} / \mathrm{H}]_{\mathrm{X}}=-0.71$ is found, which is about forty times higher than the volume-averaged one. This is because $\mathrm{X}$-ray is mainly emitted from the metal-rich gas in the hot bubbles. Hence, one-zone models of chemical evolution might lead misleading results when applied to high redshift, forming systems and an approach allowing for a detailed treatment of metal mixing and transport appears to be necessary.

The density-weighted metallicity is well above a so-called critical metallicity, $Z_{\text {crit }} \approx 10^{-5 \pm 1} \mathrm{Z}_{\odot}$ (Omukai 2000; Bromm et al. 2001; Schneider et al. 2002) marking the transition from a massive star-dominated formation mode to a normal (i.e. Salpeter) IMF. Thus, if the next generation of stars are born in the highest density regions, the IMF is expected to follow that of present-day galaxies, and the low-mass stars may form the metal-poor halo population (McWilliam et al. 1995; Ryan, Norris, \& Beers 1996). In addition, it is worth noting the existence of very low metallicity $([\mathrm{Fe} / \mathrm{H}] \approx-5)$, high density $\left(n_{\mathrm{g}} \sim 1 \mathrm{~cm}^{-3}\right)$ regions. Since these regions have metallicity below the critical value, the IMF could be similar to that of Pop III stars (e.g. Nakamura \& Umemura 2001). An extremely metal-poor star $([\mathrm{Fe} / \mathrm{H}] \approx-5.3)$ discovered by Christlieb et al. (2002) may be a relic of such stars. It has to be kept in mind, though, that by construction the studied galaxy formed from a gas of primordial composition. It is well known that at redshift $z=3$ at least some fraction of the IGM is polluted to metallicity levels of order $10^{-2.5} \mathrm{Z}_{\odot}$. If the galaxy formed from a well-mixed pre-enriched medium, its metallicity would be accordingly shifted to higher values, hence precluding the formation of extremely metal-poor stars. In order to elucidate such early enrichment in forming galaxies, LBGs may be optimal probes.

\section{Acknowledgements}

This work was supported in part by the Grant-in-Aid of the JSPS, 14740132. The numerical computations were carried on a massive parallel computer CP-PACS at the Center for Computational Physics, University of Tsukuba, using 1024 processors. The analysis of the simulation data was partially done on the parallel computer SPACE at Senshu university and the workstations at the National Astronomical Observatory in Japan.

\section{References}

Ajiki, M., et al. 2002, ApJ, 576, L25

Argast, D., Samland, M., Gerhard, O. E., \& Thielemann, F.-K. 2000, A\&A, 356, 873

Brandt, W. N., Hornschemeier, A. E., Schneider, D. P., Alexander, D. M., Bauer, F. E., Garmire, G. P., \& Vignali, C. 2001, ApJ, 558, L5

Bromm, V., Ferrara, A., Coppi, P. S., \& Larson, R. B. 2001, MNRAS, 328, 969

Christlieb, N. et al. 2002, Natur, 419, 904

Dawson, S., Spinrad, H., Stern, D., Dey, A., van Breugel, W., de Vries, W., \& Reuland, M. 2002, ApJ, 570, 92

Dey, A., Spinrad, H., Stern, D., Graham, J. R., \& Chaffee, F. H. 1998, ApJ, 498, L93

Ferguson, H. C., Dickinson, M., \& Papovich, C. 2002, ApJ, 569, L65

Ferrara, A., Pettini, M., \& Shchekinov, Y. 2000, MNRAS, 319, 539

Fujita, S. S., et al. 2003, AJ, 125, 13

Giavalisco, M. 2002, ARA\&A, 40, 579

Giavalisco, M., Steidel, C. C., \& Macchetto, F. D. 1996, ApJ, 470,189 
Hu, E. M., Cowie, L. L., McMahon, R. G., Capak, P., Iwamuro, F., Kneib, J.-P., Maihara, T., \& Motohara, K. 2002, ApJ, 568, L75 Kodaira, K., et al. 2003, PASJ, 55, L17

McWilliam, A., Preston, G. W., Sneden, C., \& Searle, L. 1995, AJ, 109,2757

Mori, M., Ferrara, A., \& Madau, P. 2002, ApJ, 571, 40

Mori, M., Yoshii, Y., \& Nomoto, K. 1999, ApJ, 511, 585

Mori, M., Yoshii, Y., Tsujimoto, T., \& Nomoto, K. 1997, ApJ, 478, L21

Nakamura, F., \& Umemura, M. 2001, ApJ, 548, 19

Nandra, K., Mushotzky, R. F., Arnaud, K., Steidel, C. C., Adelberger, K. L., Gardner, J. P., Teplitz, H. I., \& Windhorst, R. A. 2002, ApJ, 576, 625

Navarro, J. F., Frenk, C. S., \& White, S. D. M. 1997, ApJ, 490, 493

Omukai, K. 2000, ApJ, 534, 809

Papovich, C., Dickinson, M., \& Ferguson, H. C. 2001, ApJ, 559, 620

Pei, Y. C. 1992, ApJ, 395, 130

Pettini, M., Shapley, A. E., Steidel, C. C., Cuby, J., Dickinson, M., Moorwood, A. F. M., Adelberger, K. L., \& Giavalisco, M. 2001, ApJ, 554, 981

Rhoads, J. E., Malhotra, S., Dey, A., Stern, D., Spinrad, H., \& Jannuzi, B. T. 2000, ApJ, 545, 85
Ryan, S. G., Norris, J. E., \& Beers, T. C. 1996, ApJ, 471, 254 Shapley, A. E., Steidel, C. C., Adelberger, K. L., Dickinson, M., Giavalisco, M., \& Pettini, M. 2001, ApJ, 562, 95

Shapley, A. E., Steidel, C. C., Pettini, M., \& Adelberger, K. L. 2003, ApJ, 588, 65

Schneider, R., Ferrara, A., Natarajan, P., \& Omukai, K. 2002, ApJ, 571,30

Steidel, C. C., Adelberger, K. L., Shapley, A. E., Pettini, M., Dickinson, M., \& Giavalisco, M. 2000, ApJ, 532, 170

Steidel, C. C., Adelberger, K. L., Giavalisco, M., Dickinson, M., \& Pettini, M. 1999, ApJ, 519, 1

Steidel, C. C., Giavalisco, M., Dickinson, M., \& Adelberger, K. L. 1996, AJ, 112, 352

Sutherland, R. S., \& Dopita, M. A. 1993, ApJS, 88, 253

Teplitz, H. I., et al. 2000, ApJ, 533, L65

Tsujimoto, T., \& Shigeyama, T. 1998, ApJ, 508, L151

Tsujimoto, T., Nomoto, K., Yoshii, Y., Hoshimoto, M., Yanagida, S., \& Thielemann, F.-K. 1995, MNRAS, 277, 945

Weymann, R. J., Stern, D., Bunker, A., Spinrad, H., Chaffee, F. H., Thompson, R. I., \& Storrie-Lombardi, L. J. 1998, ApJ, 505, L95 